\title{
High-frequency ultrasonography (20 MHz) in circumscribed palmar hypokeratosis - new observations
}

\author{
Adriana Polańska ${ }^{1}$, Monika Bowszyc-Dmochowska ${ }^{2}$, Ryszard Żaba ${ }^{1}$, Zygmunt Adamski ${ }^{2}$, \\ Jakub Pazdrowski ${ }^{3}$, Aleksandra Dańczak-Pazdrowska²
}

\author{
${ }^{1}$ Department of Dermatology and Venereology, Poznan University of Medical Sciences, \\ Poznan, Poland \\ ${ }^{2}$ Department of Dermatology, Poznan University of Medical Sciences, Poznan, Poland \\ ${ }^{3}$ Department of Head and Neck Surgery, Greater Poland Cancer Centre, \\ Poznan University of Medical Sciences, Poznan, Poland
}

Submitted: 11 September 2014

Accepted: 1 December 2014

Arch Med Sci 2016; 12, 6: 1389-1391

DOI: 10.5114 /aoms.2016.62920

Copyright (C 2016 Termedia \& Banach

The term circumscribed palmo-plantar hypokeratosis was introduced by Perez et al. in 2002 to describe a new entity that usually appears within the palms and soles and is characterized as an asymptomatic erythematous plaque with localized loss of the horny layer [1]. The predilection sites for this disease are the palms, typically within the thenar or hypothenar eminencies. Less frequently the hypokeratosis is located within the medial portion of the sole and, interestingly, there has been only one report on nonacral localization, that is within the chest of a 63-year-old man $[2,3]$. This rare condition affects mostly middle aged and elderly women, and its etiopathogenesis still remains a matter of debate [1-3]. Until 2014 according to the PubMed database there were approximately 70 case reports in the literature regarding this disease, but none of them came from central Europe. The diagnosis of this disease requires confirmation in histopathological examination. However, $20 \mathrm{MHz}$ ultrasonography (high-frequency ultrasonography - HF-USG) seems to be additionally useful, because it allows for noninvasive observation of its unique characteristics.

We present a case of circumscribed palmar hypokeratosis diagnosed in a 63-year-old woman in whom we performed HF-USG.

A 63-year-old woman was admitted to the outpatient clinic due to an asymptomatic lesion located on the thenar eminence of the left hand that has been observed for 10 years.

In physical examination a $2.7 \mathrm{~cm} \times 2.3 \mathrm{~cm}$ solitary erythematous patch within the thenar eminence of the left hand was observed (Figure 1). The lesion was slightly depressed, with a well-demarcated raised border. The patient did not associate the presence of the condition with any history of trauma or other possible triggering factors. Previous topical treatment with glucocorticosteroids was ineffective.

We performed $20 \mathrm{MHz}$ B-mode HF-USG (Dermascan C ver. 3, Cortex Technology, Hadsund, Denmark). This instrument operates at a frequency of $20 \mathrm{MHz}$, with a resolution of $60 \mu \mathrm{m} \times 200 \mu \mathrm{m}$ (axial $\times$ lateral) and approximately $15-\mathrm{mm}$ penetration. At the border of lesional and nonlesional skin HF-USG revealed abrupt loss of double entrance echo with only single entrance echo within all affected skin, below which a subepi-

\author{
Corresponding author: \\ Adriana Polańska MD, PhD \\ Department of Dermatology \\ Poznan University \\ of Medical Sciences \\ 49 Przybyszewskiego St \\ 60-355 Poznan, Poland \\ Phone: +48 516123858 \\ E-mail: adriana-polanska@ \\ wp.pl
}




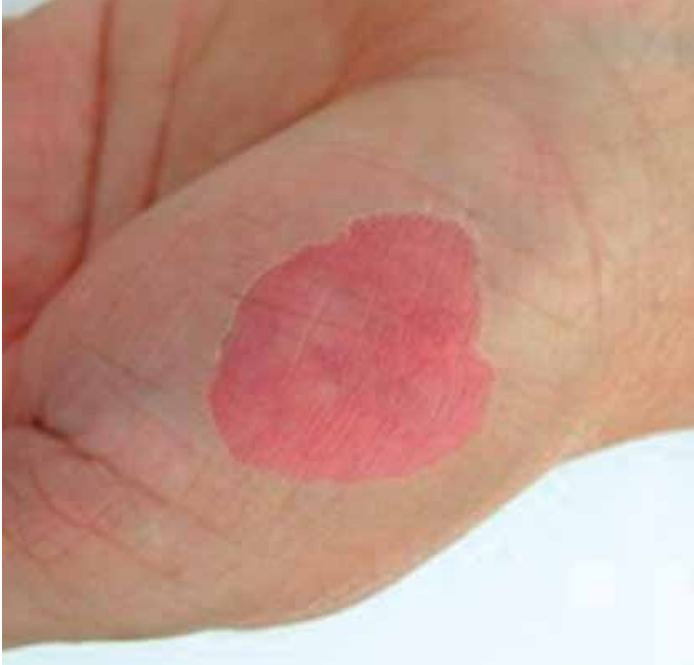

Figure 1. Erythematous path within thenar eminence

dermal low echogenic band (SLEB) was visualized. The border between lesional and nonlesional skin was sharply accentuated (Figure 2).

The biopsy for histological examination taken from the border of the lesion revealed abrupt thinning of the horny layer, up to one third of its original thickness, with a sharp stair-like edge. The epidermis and dermis below the depression of the stratum corneum were normal (Figure 3).

To our knowledge the case discussed in this paper is probably the first in which HF-USG was used as a diagnostic method. We describe the unique abrupt loss of double entrance echo with only single entrance echo within affected skin, a characteristic feature of palmar hypokeratosis, which was not reported in previous literature and corresponds to the histopathological findings.

In 2002 hypokeratosis was classified as a benign clonal epidermal malformation [1]. Although until now several new descriptions have been reported, still its etiopathogenesis is unknown. One

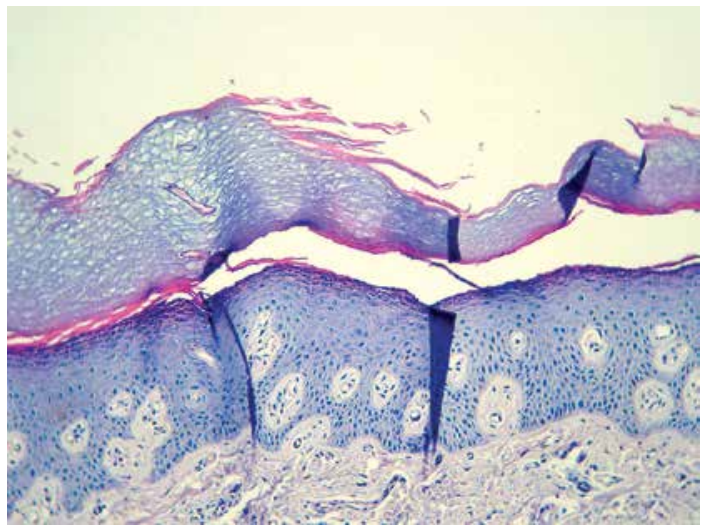

Figure 3. Abrupt thinning of the orthokeratotic stratum corneum with sharp stair-like edge over otherwise normal epidermis and dermis $(\mathrm{H}+\mathrm{E}$, original objective magnification 20x)

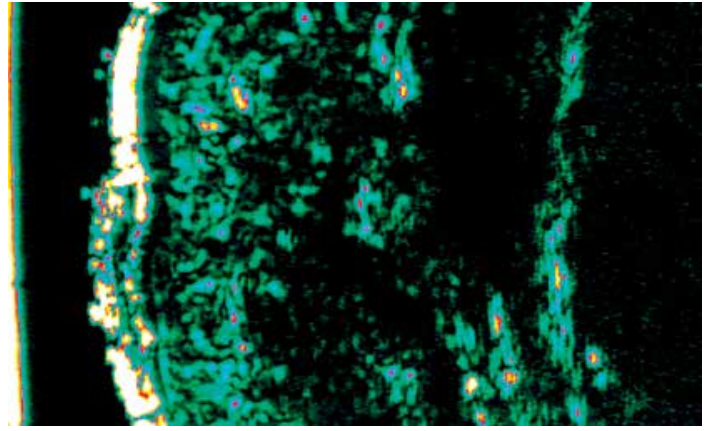

Figure 2. $20 \mathrm{MHz}$ skin USG of hypokeratosis - at the border of lesional and nonlesional skin HFUSG abrupt loss of double entrance echo with only single entrance echo within all affected skin is observed, with the presence of subepidermal low echogenic band (SLEB)

theory suggests that acral hypokeratosis is a primary keratinizing disorder [4]. Another hypothesis is related to the defect in epidermal structure with abnormalities in attachment structures, impaired intercellular cohesion and early detachment of corneocytes [5]. Another research group postulated deregulation in the desquamation process [6]. An interesting etiopathogenic factor may be linked to human papillomavirus infection, and hypokeratosis has also been thought to be the result of trauma [7].

Definitive diagnosis of acral hypokeratosis is based on histopathological examination. The biopsy, which should include the edge of the lesion, always reveals abrupt reduction of the stratum corneum in the lesional skin clinically associated with a reddish depression. The biopsy is by the invasive method; thus HF-USG, which presents with an image characteristic for hypokeratosis, may serve as an initial, additional tool, which may speed up the diagnosis. In HF-USG we observed the lack of a typical image for palmar skin. There was no sign of a double entrance echo within lesional skin. The HF-USG of non-affected palmar skin revealed a three-layered structure $[8,9]$. The first layer is known as an entrance echo (a strongly echogenic band), below which is a dermis (a layer rich in echoes of the scattered reflections) and then very thin subcutaneous tissue - a layer of low echogenicity with well-reflecting wave compartments. The entrance echo in the palms, due to the thick stratum corneum, is formed of two high echogenic bands (known as a double entrance echo), which is related to the transition between the weakly hydrated stratum corneum and layers of living epidermis, which are rich in water [8]. Additionally, at the border of lesional and nonlesional skin, abrupt loss of the double entrance echo is detected, similarly to the histopathological result (stair-like edge). We also found SLEB, which may be an indicator of infiltration with inflammatory 
or neoplastic cells, or papillary edema, as well as being a sign of photodamaged skin $[9,10]$. In our case, similarly to previous reports, there was no sign of inflammation or elastosis of papillary dermis within the area of hypokeratosis, which could explain the observation of SLEB. The SLEB of hypokeratosis is an unexpected phenomenon and may be, to some extent, related to the slightly dilated papillary vessels.

In conclusion, we would like to stress that the observed abrupt loss of the double entrance echo is in close correlation to the main histological feature of hypokeratosis - the stair-like edge. However, further studies including a greater number of patients will be needed to confirm the results. What is more, because HF-USG is a noninvasive method, it can be used to assess the efficiency of different types of therapy.

\section{Conflict of interest}

The authors declare no conflict of interest.

\section{References}

1. Pérez A, Rütten A, Gold R, et al Circumscribed palmar or plantar hypokeratosis: a distinctive epidermal malformation of the palms or soles. J Am Acad Dermatol 2002; 47: 21-7.

2. Groysman T, Rothfleisch J, Baldassano MF. Circumscribed palmar or plantar hypokeratosis: first report on a nonacral site with unique histologic features. Am J Dermatopathol 2013; 35: 491-3.

3. Barry Cl, Glusac EJ, Kashgarian M, Gwin K, McNiff JM. Circumscribed palmar hypokeratosis: two cases and a review of the literature. J Cutan Pathol 2008; 35: 484-7.

4. Ishiko A, Dekio I, Fujimoto A, et al. Abnormal keratin expression in circumscribed palmar hypokeratosis. J Am Acad Dermatol 2007; 57: 285-91.

5. Yamamoto O, Yoshida Y, Adachi K, Yamada N. Abnormal cell attachment in circumscribed palmar hypokeratosis: ultrastructural observations. Br J Dermatol 2011; 164: 1395-6.

6. Tanioka M, Miyagawa-Hayashino A, Manabe T, Toichi E, Miyachi Y, Takahashi K. Circumscribed palmo-plantar hypokeratosis: a disease with two subtypes. J Invest Dermatol 2009; 129: 1045-7.

7. Riveiro-Falkenbach E, Rodríguez-Peralto JL. Circumscribed palmar hypokeratosis associated to a burn scar. Am J Dermatopathol 2013; 35: 246-7.

8. Jemec GB, Gniadecka M, Ulrich J. Ultrasound in dermatology. Part I. High frequency ultrasound. Eur J Dermatol 2000; 10: 497-2.

9. Jasaitiene D, Valiukeviciene S, Linkeviciute G, et al. Principles of high-frequency ultrasonography for investigation of skin pathology. J Eur Acad Dermatol Venereol 2011; 25: 375-82.

10. Polańska A, Dańczak-Pazdrowska A, Silny W, et al. Comparison between high-frequency ultrasonography (Dermascan C, version 3) and histopathology in atopic dermatitis. Skin Res Technol 2013; 19: 432-7. 